\title{
Investigation and Analysis of International Demands for Development of Acupuncture-moxibustion Technical Specifications and General Rules for Drafting
}

Jin Huo

China Academy of Chinese Medical Sciences Institute of Acupuncture and Moxibustion

Zhong-Jie Chen

China Academy of Chinese Medical Sciences Institute of Acupuncture and Moxibustion

Qi Gao

China Academy of Chinese Medical Sciences Institute of Acupuncture and Moxibustion

Xiao-Yu Wang

China Academy of Chinese Medical Sciences Institute of Acupuncture and Moxibustion

\section{Yi Zhang}

China Academy of Chinese Medical Sciences Institute of Acupuncture and Moxibustion Jing Hu

China Academy of Chinese Medical Sciences Institute of Acupuncture and Moxibustion

\section{Xiao-Dong Wu}

China Academy of Chinese Medical Sciences Institute of Acupuncture and Moxibustion

Jingjing WANG ( $\square$ wjj751@sina.com )

China Academy of Chinese Medical Sciences Institute of Acupuncture and Moxibustion https://orcid.org/0000-0002-0361-2550

\section{Research}

Keywords: International standards, Acupuncture-moxbustion technique specifications, General rules, Surveys

Posted Date: April 1st, 2020

DOl: https://doi.org/10.21203/rs.3.rs-19777/v1

License: (9) This work is licensed under a Creative Commons Attribution 4.0 International License. Read Full License 


\section{Abstract}

Background To investigate and analysis of international demands of Chinese and international experts for the development of A cupuncture-moxibustion t echnical s pecifications and $\mathrm{G}$ eneral $\mathrm{r}$ ules for d rafting.

Methods The target of the study was selected from experts in the field of acupuncture-moxibustion standardization and from some acupuncture-moxibustion experts. Questionnaire was developed through Questionnaire Star (wjx.cn). 187 links of target-oriented online questionnaires were sent via E-mail. 102 valid questionnaires returned. Excel was adopted to establish database and analyze the statistics.

Results $98.04 \%$ of the experts hold that it is necessary to develop the international standard, the Technical Specification of Acupuncture-moxibustion: General Rules for Drafting. A survey on the scope of the existing standards shows that demands of experts inside and outside Chinese mainland are slightly different. Standards of the Chinese mainland highlight commonality. While countries/regions outside the Chinese mainland emphasize on individuality. All experts (inside and outside the Chinese mainland) reach the consensus that importance shall be attached to the common issues concerning acupuncture-moxibustion application process. However, wide discrepancy is found in 3 aspects in terms of 'relevant terminology', 'pre-operative preparation' and 'adverse reactions and contraindications'.

Conclusions There is a great international demand for developing the international standard of the Technical Specification of Acupuncture-moxibustion: General Rules for Drafting . Ensuring the accuracy of the common problems during the development process, demands of different countries/regions shall be fully considered. Effort shall be given in balance between refining the common problems and meeting the special demands, so as to enhance the international compatibility and applicability.

\section{Background}

At present, in the field of technical operation of acupuncture-moxibustion, there are only two international standards, the Moxibustion Manipulations ${ }^{[1]}$ and Scalp Acupuncture Manipulation ${ }^{[2]}$, which were issued by the World Federation of Acupuncture-Moxibustion Societies (WFAS) in 2013. The development of a series of international standards for acupuncture-moxibustion technical operations will effectively promote the safe and effective use of acupuncture-moxibustion in the world, and will promote the global development of Chinese Medicine.

In the current Chinese national standards, the series of Technical Specification for Acupuncture has been issued in batches since 2008 and 22 terms have been issued till now ${ }^{[3]}$. Technical Specification of Acupuncture-moxibustion: General Rules for Drafting ${ }^{[4]}$ (herein after refer to as the General Rules) was issued in 2017 to regulate the series of national standards. In order to promote the international standardization of acupuncture-moxibustion, this study conducted a questionnaire survey on the international demands for Technical Specification of Acupuncture-moxibustion: General Rules for drafting. 


\section{Methods}

\section{Participants}

Domestic and international experts in the field of acupuncture standardization and some foreign acupuncture-moxibustion experts were selected as the participants, including all the experts in the expert committee of this program, members of the Standardization Working Committee of WFAS(SC-WFAS), international experts in International Organization for Standardization/ Technical Committee of Chinese Medicine (ISO/TC249), main drafters of current acupuncture standards, members of national technical committee on Acupuncture-Moxibustion of Standardization Administration of China (SAC/TC475), members of Standardization Work Committee of Chinese Acupuncture-Moxibustion Society, and acupuncture experts engaged in clinical work abroad for a long time.

\section{Investigation method}

WFAS have no uniform regulations of developing standards. Questions concerning the content of the General Rules in this survey were formulated with the relevant provisions of ISO ${ }^{[5]}$ and WHO ${ }^{[6]}$. The online questionnaires in Chinese and English were made on the Internet platform of Questionnaire Star (www.wjx.cn). The main questions of the questionnaires were choices and order sorting questions. Supplementary filling options were set for multiple-choice questions. The contents of the questionnaires focus on the international demands of Technical Specification of Acupuncture-moxibustion: General Rules for drafting. There were 20 basic research questions for experts in the Chinese mainland. In order to know international demands better, 8 more questions were added to the questionnaires for areas outside the Chinese mainland. Questionnaire links were directly sent via e-mail. The English and Chinese versions of the General Rules were sent as attachments to the experts for reference at the same time. A 7-day due from the sending day was set. Two rounds of response reminders were sent as well via email, wechat, SMS and other channels.

\section{Results}

187 online questionnaire were sent, out of which 102 valid survey questionnaires were collected $\$ the effective response rate is $54.55 \%$. Respondents are from 18 countries and regions ( 11 countries in Europe, 3 countries in the Americas, 3 countries/regions in Asia, and 1 country in Oceania). 62 respondents are from the Chinese mainland and 40 outside the Chinese mainland. Their working experience in acupuncture is $21.2 \pm 14.3$ years.

\section{Legal perspective}

Regarding the legality of acupuncture, $97.50 \%$ of the experts surveyed outside the Chinese mainland replied that their countries/regions acknowledged the legal status of acupuncture-moxibustion (39/40). One French respondent chose 'illegality'. However, other French respondents who were surveyed in the same period all chose 'legality'. Regarding the question of 'whether there is acupuncture-related 
legislation', there were experts from 8 countries/ regions (the United States, Canada, Australia, Switzerland, Romania, Russia, South Korea, and Hong Kong) responded unanimously to 'yes'. Experts of

3 countries responded 'no' (Sweden, Norway, Brazil). Experts from the other 6 countries responded inconsistently (Germany, Italy, Spain, the United Kingdom, France, the Netherlands). 92.50\% experts believe that the development of the standard of Technical Specification of Acupuncture-moxibustion: General Rules for drafting will help them to improve the legal status of acupuncture-moxibustion in their countries/regions and promote acupuncture-moxibustion legislation.

\section{Perspective on payment for acupuncture-moxibustion}

In the response to the question 'Does the national public medical insurance cover the cost of acupuncture-moxibustion treatment?', of the 40 experts, only $15.00 \%(6 / 40)$ chose 'it is paid', $17.50 \%$ (7 /40) chose 'partial paid', and the remaining $67.50 \%$ (27/40) selected 'it is not paid'. In the response to the question 'Does private medical insurance cover the cost of acupuncture-moxibustion treatment', $40.00 \%$ (16/40) chose 'it is paid', $42.50 \%$ ( $17 / 40)$ chose 'partial paid', and $17.50 \%(7 / 40)$ chose 'it is not paid'. $90.00 \%$ (36/40) of experts believe that the publishing of the series of international General Rules will help expand the scope and proportion of acupuncture-moxibustion payments in the medical insurance system of their countries/regions.

\section{International application of common acupuncture-moxibustion techniques}

As shown in table 1, the application of 24 acupuncture techniques which are covered by the existing Chinese standards in countries/regions outside the Chinese mainland are investigated. More than $50 \%$ of the acupuncture-moxibustion techniques are widely used. Most of them are conventional acupuncture techniques with relatively high international popularity. Prioritization is done on demands for developing international standards for different acupuncture-moxibustion technical specifications. The average comprehensive score is calculated by the sorting integral method. Acupuncture-moxibustion techniques with an average score over 10 points are 'filiform needling method' (22.20), 'moxibustion' (18.37), 'electroacupuncture' (17.23), 'cupping therapy'(13.85) and 'ear acupuncture therapy'(11.36). These 5 acupuncture-moxibustion techniques are widely used internationally and have a high popularity. Thus, it is concluded that developing priorities shall be given to international standards of these acupuncturemoxibustion techniques.

\section{The existing national General Rules for drafting}

The awareness rate of the existing General Rules is $71.57 \%$ of 102 experts, among which $52.50 \%$ are outside the Chinese mainland, $83.87 \%$ are inside the Chinese mainland. $64.71 \%$ of the total number of the experts have consulted or received relevant training, among which $50.00 \%$ are outside the Chinese mainland and $74.19 \%$ inside the Chinese mainland. Utilization rate of the General Rules is $72.55 \%$, among which $57.50 \%$ are outside the Chinese mainland and $82.26 \%$ inside the Chinese mainland.

$98.04 \% \bigotimes 100 / 102 \rrbracket$ of the total number of the experts believe that it is necessary to develop the international standard of the General Rules. $79.41 \%$ (81/102) considered that the existing General Rules 
need to be revised. As shown in Figure 1, differences in the proportion of options on 'what need to be revised' can be found in experts inside and outside Chinese mainland. The number of experts outside the Chinese mainland are higher than that of the experts inside the Chinese mainland in the option 'The expression is not clear enough'. While the number of experts inside the Chinese mainland is significantly higher than that outside the Chinese mainland in the option 'Not practical enough'.

\section{Demands for the scope of the international General Rules for drafting}

As shown in Figure 2, demands of experts inside and outside the Chinese mainland are slightly different in term of scope. A greater difference is found in the option of 'cover common issues in manipulating various kinds of acupuncture-moxibustion' (52.50\% outside the Chinese mainland, $91.94 \%$ inside the Chinese mainland). Experts in the Chinese mainland want to highlight commonalities while experts outside the Chinese mainland want to retain more specialties. Some experts also comment that the item of 'basic requirement' should be adapted according to the situations in different countries. The work flow for the WFAS standard should be clarified and the text structure and abbreviations should be unified in processing the English version.

\section{Common issues of acupuncture-moxibustion operations}

All experts agree that common issues should be emphasized in acupuncture-moxibustion application process. However, in Figure 3, their opinions differ greatly in terms of 'relevant terminology', 'pre-operative preparation' and 'adverse reactions and contraindications'. Experts outside the Chinese mainland paid less attention to the above-mentioned issues while experts inside the Chinese mainland considered them to be very important. In addition, some experts inside the Chinese mainland also added issues such as 'the tolerance definition for acupuncture-moxibustion techniques in different countries' and 'compatibility and adaptability in different countries'.

\section{Discussion}

The survey covers a wide range of countries/regions. Participants of the survey are qualified in the professional background of international standards development. According to the survey, many countries in the world have demands for acupuncture-moxibustion legislation. However, due to the insufficient recognized international standards of acupuncture-moxibustion techniques, many countries lack the indispensable technical supports for their acupuncture-moxibustion legislation. As shown in the previous studies of WFAS [7], the legislative process of acupuncture-moxibustion are various in different countries and is influenced by many factors, namely the professionalism of the practitioners in their countries, the popularity of acupuncture-moxibustion in these countries, the importance attached by their governments and political parties as well as the international environment. It is a long and difficult road from aware of the demand for laws and regulations to the action of legislation and further to the enforcement. The development of a series of international specifications will be helpful in promoting acupuncture-moxibustion legislation at the international level, improving the legal status of acupuncture- 
moxibustion, increasing the proportion of acupuncture-moxibustion payments in the medical insurance system and promoting the popularization and spread of acupuncture-moxibustion techniques. Thus, there is a great international demand of the development and this program is designed to meet such international demands.

Standard is a generalization of individuation. It aims at seeking common ground by sacrificing individuality. However, in the development of international standards, attention shall not only be paid to commonality but also individuality. To ensure the accuracy of the common problems, emphasis shall be given to keeping a balance between refining the common problems and meeting individual demands of different countries. Thus, the international compatibility and applicability of this standard can be improved and the safe and effective use of acupuncture-moxibustion can be promoted internationally.

Experts who did not respond to the questionnaire are mainly international experts of ISO/TC249, particularly in the countries of Asia, and the response rate is only $21.05 \%(8 / 38)$. Followed by SC-WFAS members from countries/regions outside the Chinese mainland. Their response rate is only $28.57 \%$ (4/14). The low participation rate of international experts in the two major international organizations closely related to the international standardization of acupuncture-moxibustion is the greatest pity of this investigation. Since it is lack of experts' opinions of these two organizations, the sample size of this round of the survey is relatively small[ ${ }^{[8]}$ and the global representativeness of the results of this survey seems to be not sufficient enough ${ }^{[9]}$.

\section{Conclusion}

In summary, through this round of demand survey, the huge international demand for the development of the International Standards for Technical Specification of Acupuncture-moxibustion: General Rules for drafting has been clarified, indicating the direction for the next step of the development.

\section{Abbreviations}

WFAS: World Federation of Acupuncture-Moxibustion Societies; SC-WFAS: Standardization Working Committee of WFAS; ISO/TC: International Organization for Standardization/ Technical Committee of Chinese Medicine; SAC/TC: National Technical Committee on Acupuncture-Moxibustion of Standardization Administration of China; WHO: World Health Organization

\section{Declarations}

\section{Ethics approval and consent to participate}

Not applicable.

\section{Consent for publication}


Not applicable.

\section{Availability of data and materials}

All data generated or analysed during this study are included in this published article.

\section{Competing interests}

The authors declare that they have no competing interests.

\section{Funding}

National Key R \& D Projects (No.2019 YFC1712200) Project (No.2019 YFC 1712202) .

\section{Authors' contributions}

J.Huo and ZJC participated in the design of the questionnaire. JJW is the project leader, in charge of the research direction, contacted experts via email to invite them to participate in the investigation. XYW did the data search and extracted data. J.Hu performed all statistical analyses. J.Huo wrote the paper, which was reviewed and edited by $Y Z, Q G$ and JJW. As the main draftsman of the current national standard, XDW provided the background information. All authors read and approved the final version of the manuscript accepted for publication.

\section{Acknowledgements}

Not applicable.

\section{Authors' information}

Institute of Acupuncture and Moxibustion, China Academy of Chinese Medical Sciences.

\section{References}

1.World Federation of Acupuncture-Moxibustion Societies(WFAS).WFAS STANDARD 003:2013.China Press of Traditional Chinese Medicine.

2.World Federation of Acupuncture-Moxibustion Societies(WFAS).WFAS STANDARD 004:2013.China Press of Traditional Chinese Medicine.

3.Ma XT, Zhai W, Tan YQ, etal. Literature Study on the Use Situation of Acupuncture-moxibustion Operation Standardization. Shanghai J Acu-mox .2016;35:751-53.

4.China National Standardization Administration.GB/T 33416-2016:Specification of manipulations of acupuncture and moxibustion-General Rules for drafting. 
5.International Organization for Standardization(ISO). ISO guidelines, Part 2, Principles and rules for the structure and drafting of ISO and IEC documents2018;Available at

https://isotc.iso.org/livelink/livelink?func=\|l\&objld=4230456\&objAction=browse\&sort=subtype.

6. World Health Organization(WHO).WHO handbook for guideline development, 2nd ed 2014;Available at https://apps.who.int/iris/handle/10665/14714.

7.Yang YY, Shen ZX, Wu ZC, etal. Strategy programming for acupuncture development along One-BeltOne-Road countries. Chinese Acupuncture \&Moxibustion.2017;37:343-48.

8.Thomas V. Perneger, Delphine S. Courvoisier, Patricia M. Hudelson, etal. Sample size for pre-tests of questionnaires. Quality of Life Research.2015;24:147-51.

9.Barbara Kitchenham, Shari Lawrence Pfleeger. Principles of survey research part 5囚Populations and Samples. ACM SIGSOFT Software Engineering Notes.2002;3: 17-20.

\section{Tables}

Table 1 International application of common acupuncture techniques

\begin{tabular}{ccc}
\hline Technique & Proportion of applications $\square$ \% & $\begin{array}{c}\text { Average comprehensive score } \\
\text { (points) }\end{array}$ \\
\hline moxibustion & 97.50 & 18.37 \\
cupping therapy & 95.00 & 13.85 \\
filiform needling method & 92.50 & 22.20 \\
electro-acupuncture & 92.50 & 17.23 \\
ear acupuncture therapy & 87.50 & 11.36 \\
scalp acupuncture therapy & 77.50 & 9.52 \\
scraping therapy & 75.00 & 6.28 \\
abdominal acupuncture therapy & 75.00 & 4.41 \\
wrist-ankle acupuncture therapy & 70.00 & 4.33 \\
cutaneous needle therapy & & 3.30 \\
intradermal needle therapy & 67.50 & 4.57 \\
plaster applied to a point therapy & 57.50 & 3.54 \\
three-edged needle therapy & 57.50 & 5.97 \\
nose acupuncture therapy & 50.00 & 1.05 \\
umbilicus needle therapy & 50.00 & 1.34 \\
eye acupuncture therapy & 40.00 & 1.47 \\
oral and lip acupuncture therapy & 37.50 & 1.12 \\
fire needling therapy & 35.00 & 5.61 \\
thread-imbedding therapy & 27.50 & 3.86 \\
point injection therapy & 27.50 & 4.08 \\
awn-like needle therapy & 25.00 & 4.15 \\
long-round needle therapy & 17.50 & 1.02 \\
spoon-like needle therapy & 15.00 & 1.09 \\
acupotomy therapy & 12.50 & 3.24 \\
\hline
\end{tabular}

\section{Figures}




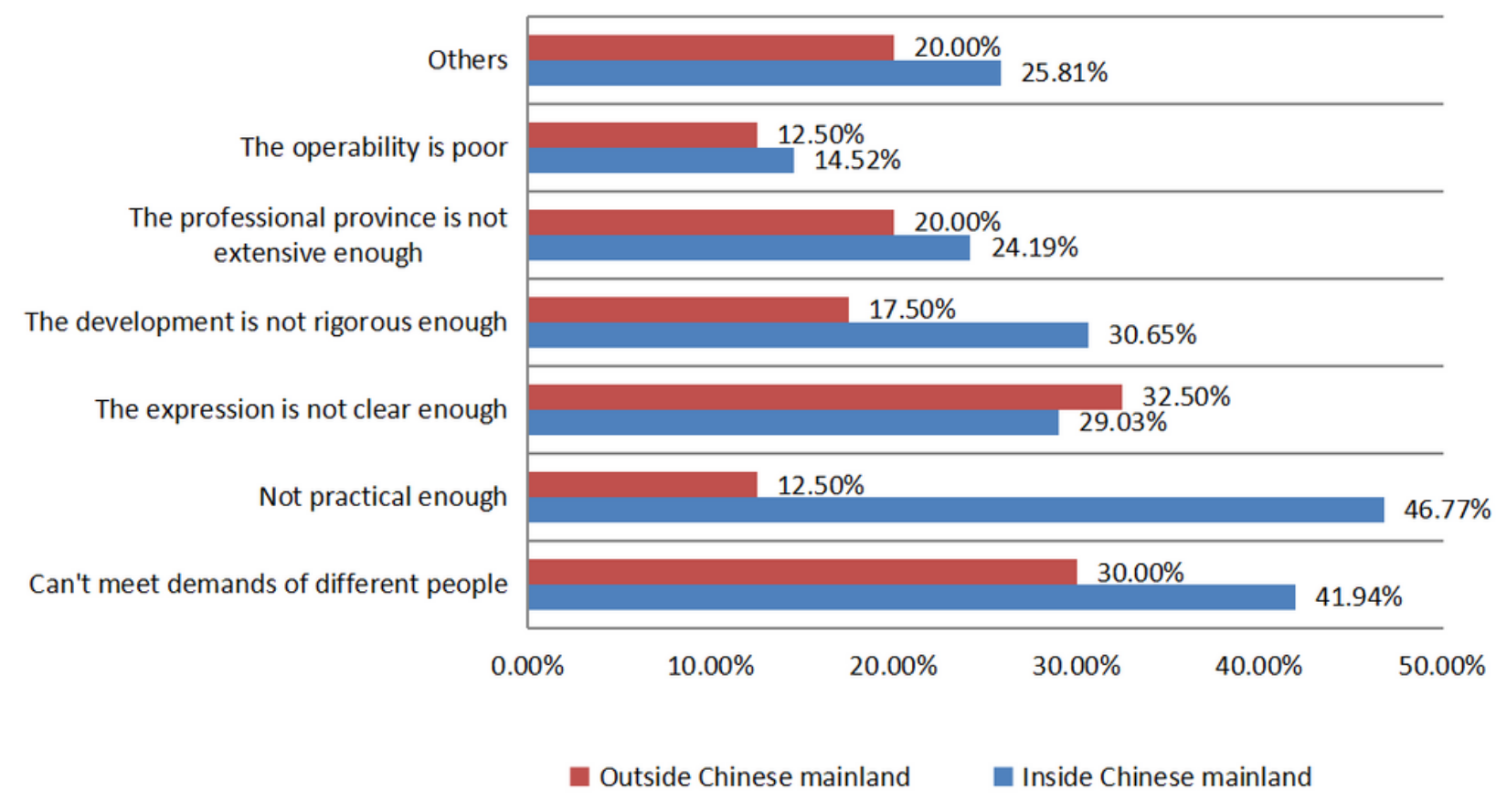

\section{Figure 1}

A caption was omitted by the authors in this version of the paper.

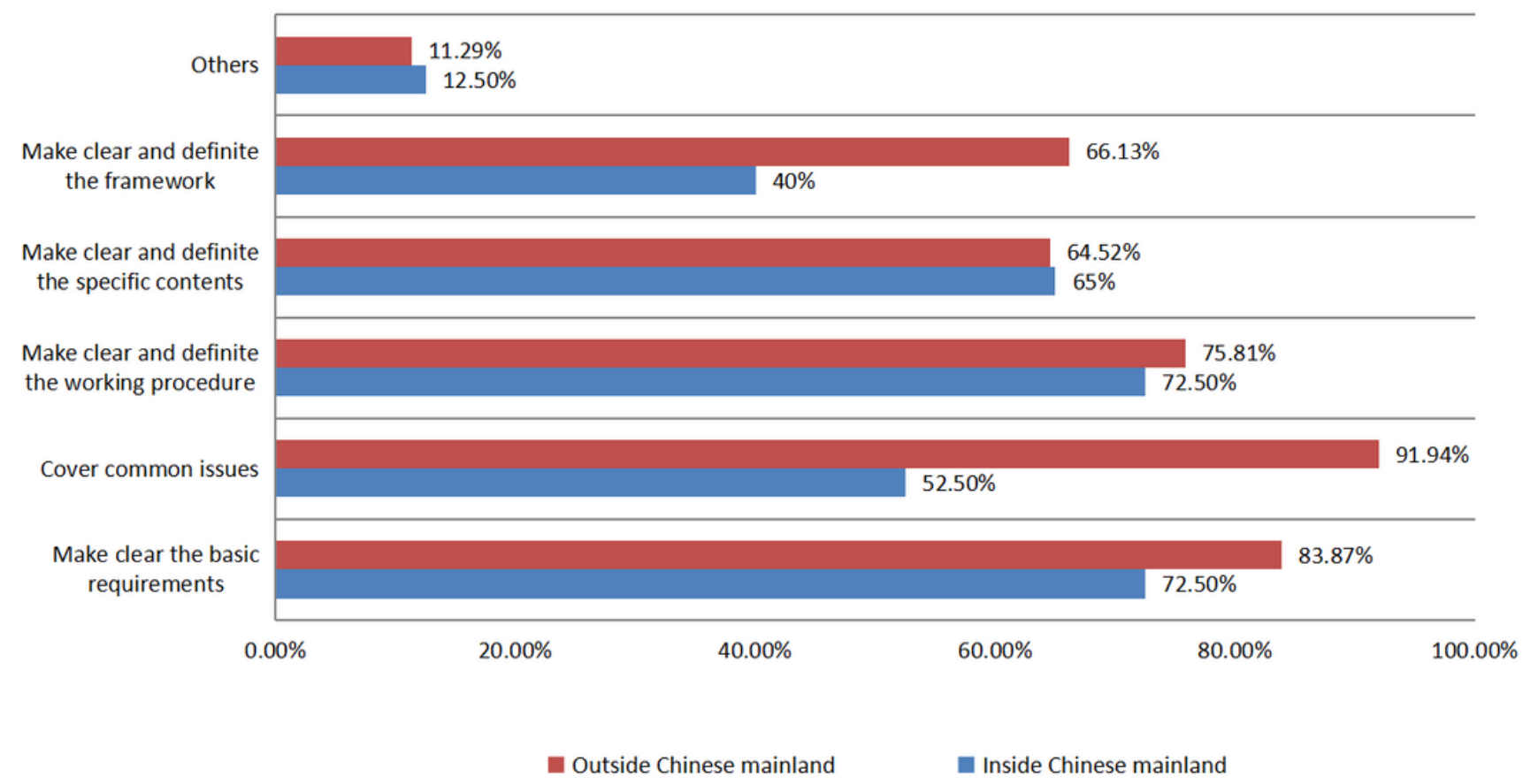

Figure 2

A caption was omitted by the authors in this version of the paper. 


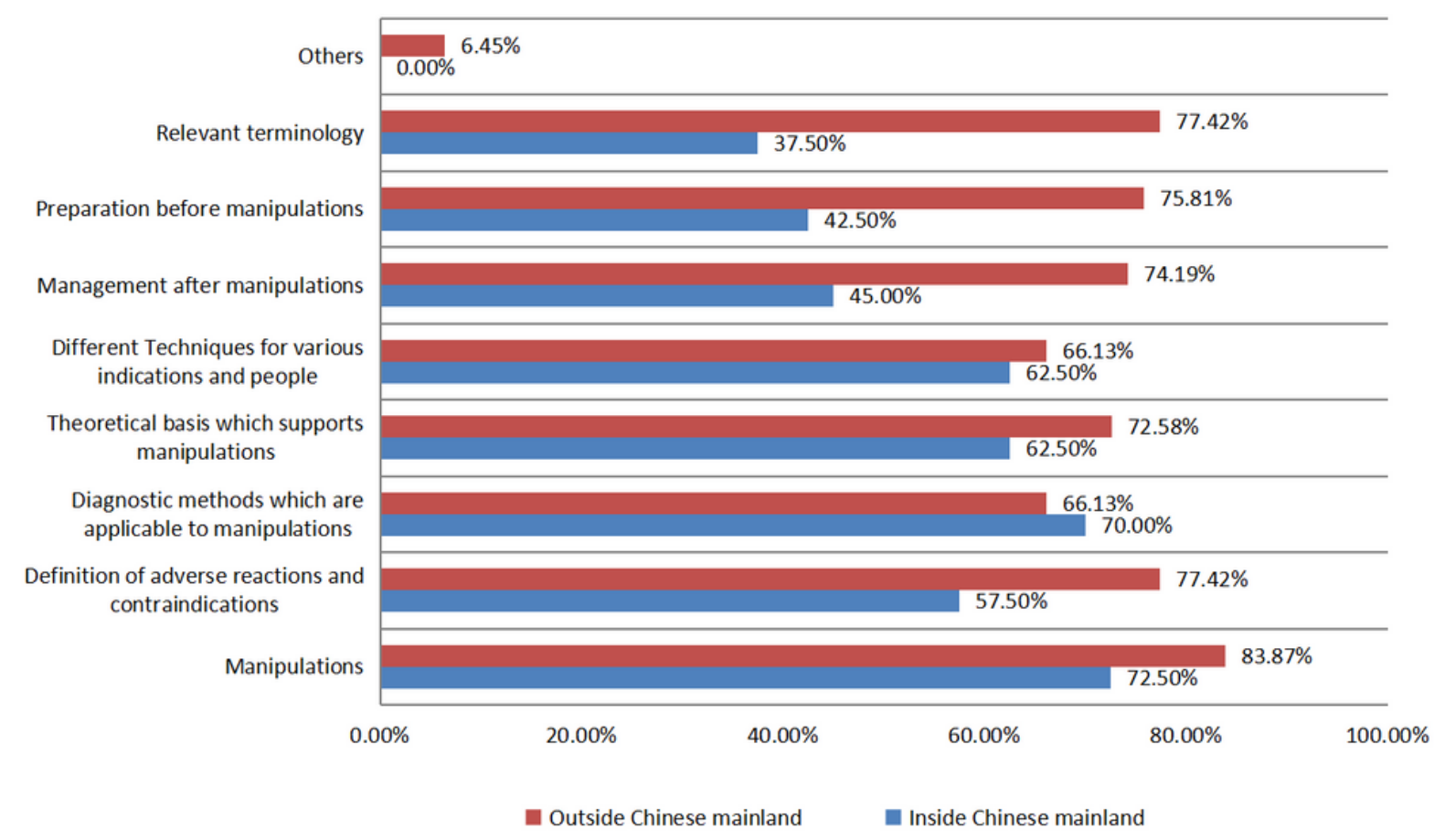

Figure 3

A caption was omitted by the authors in this version of the paper. 